\title{
Micro-architectural changes in cancellous bone differ in female and male C57BL/6 mice with high-fat diet-induced low bone mineral density
}

\author{
Jyoti Gautam ${ }^{1}$, Dharmendra Choudhary ${ }^{1}$, Vikram Khedgikar ${ }^{1}$, Priyanka Kushwaha ${ }^{1}$, \\ Ravi Shankar Singh ${ }^{2}$, Divya Singh ${ }^{1}$, Swasti Tiwari ${ }^{2}$ and Ritu Trivedi ${ }^{1}$ * \\ ${ }^{1}$ Division of Endocrinology, Council of Scientific and Industrial Research (CSIR)-Central Drug Research Institute, \\ Lucknow, India \\ ${ }^{2}$ Department of Molecular Medicine and Biotechnology, Sanjay Gandhi Post Graduate Institute of Medical Sciences, \\ Lucknow, India \\ (Submitted 29 May 2013 - Final revision received 17 December 2013 - Accepted 19 December 2013 - First published online 10 February 2014)
}

\section{Abstract}

The relationship between fat and bone mass at distinct trabecular and cortical skeletal compartments in a high-fat diet (HFD) model was studied. For this, C57BL/6 mice were assigned to four groups of eight animals each. Two groups, each of males and females, received a standard chow diet while the remaining other two groups received the HFD for a period of 10 weeks. Male mice on the HFD were heavier and gained more weight $(15.8 \% ; P<0.05) v$. those on the control diet or when compared with the female rats fed the HFD. We observed an increased lipid profile in both males and females, with significantly higher lipid levels (about $20-25 \% ; P<0 \cdot 01$ ) in males. However, glucose intolerance was more pronounced in females than males on the HFD (about $30 \% ; P<0 \cdot 05$ ). The micro-architectural assessment of bones showed that compared with female mice on the HFD, male mice on the HFD showed more deterioration at the trabecular region. This was corroborated by plasma osteocalcin and carboxy-terminal collagen crosslinks (CTx) levels confirming greater loss in males (about $20 \% ; P<0.01)$. In both sexes cortical bone parameters and strength remained unchanged after 10 weeks of HFD treatment. The direct effect of the HFD on bone at the messenger RNA level in progenitor cells isolated from femoral bone marrow was a significantly increased expression of adipogenic marker genes $v$. osteogenic genes. Overall, the present data indicate that obesity induced by a HFD aggravates bone loss in the cancellous bone compartment, with a greater loss in males than females, although 10 weeks of HFD treatment did not alter cortical bone mass and strength in both males and females.

Key words: High-fat diet: Osteoporosis: Microcomputed tomography: C57BL/6 mice: Glucose tolerance

Osteoporosis and obesity are two disorders that share several features including genetic predisposition and common progenitor cells ${ }^{(1)}$. While genetic factors contribute to an individual's risk of developing osteoporosis and subsequent fragility fractures, modifiable factors such as nutrition can also influence bone health either positively or negatively ${ }^{(2)}$. The connection between fuel utilisation and skeletal remodelling begins in the bone marrow with lineage allocation of mesenchymal stem cells to adipocytes or osteoblasts $^{(3)}$. Mature bone cells secrete factors that influence insulin sensitivity, and fat cells synthesise cytokines that regulate osteoblast differentiation; thus, these two pathways are closely linked. Any effects beneficial to bone are mainly based on well-established positive correlations between body weight or mechanical loading conferred by body weight and bone mineral density found in animals and humans ${ }^{(4)}$. There are studies that suggest positive correlations between fat mass and bone. A study by Ma et al. ${ }^{(5)}$ has shown that in mice the femur bone adapts to obesity and exhibits improved bone strength even at the micro-structural level. Adaptation is also seen in response to running exercise, with increased trabecular density and improved three-dimensional structure $^{(5)}$. However, accumulating data also suggest that excessive fat mass is detrimental to bone ${ }^{(6,7)}$. In a study by Hsu et al. ${ }^{(8)}$ in a large cohort of a Chinese population, with a certain body weight there was a negative relationship between fat mass and bone mass. Risk of osteoporosis, osteopenia and non-spine fractures were significantly higher in subjects with higher percentage body fat, independent of body weight. The negative genetic correlation denoted by

Abbreviations: ALP, alkaline phosphatase; BMSC, bone marrow stromal/osteoblastic cells; BV/TV, bone volume/tissue volume; CTx, carboxy-terminal collagen crosslinks; HFD, high-fat diet; mRNA, messenger RNA; OCN, osteocalcin; PBM, peak bone mass; SMI, structural model index; Tb.N, trabecular number; Tb.Sp, trabecular separation.

*Corresponding author: Dr Ritu Trivedi, email ritu_trivedi@cdri.res.in 
$\rho G$ between fat mass and weight-adjusted bone mass suggests that fat and bone mass share some common genetic factors and molecular pathways that appear to have opposite effects on fat $v$. bone mass ${ }^{(9)}$.

In growing animal models, a high-fat diet (HFD) could deleteriously affect bone mineral content, structure and mechanical properties ${ }^{(10)}$. Consistent with our data, a recent study on young male mice suggests that HFD-induced obesity in growing animals may affect the total available osteoblastic cell differentiation progenitors in the bone with increasing adipogenesis. This may result in negative consequences for the bone later on in adult life ${ }^{(11)}$. These discrepancies could be explained by the different animal characteristics (sex, age and breed), the duration and the quality of the diet used. However, finally, it was the diet that could influence the results or the outcome by its ability to either induce obesity or not ${ }^{(12)}$.

Various intervention studies on murine bone structure have been carried out in ageing models that indicate that cortical thickness decreases and that the cross-sectional moment of inertia increases with increasing age. In addition, total femoral volumetric bone mineral density increases until 4 months of age and is maintained thereafter ${ }^{(13)}$. Studies on diet-induced obesity mouse models indicate that a HFD decreases cancellous bone mass but has no effect on cortical bone mass in tibia in mice ${ }^{(4)}$. One interesting study has also investigated the possible role of HFD-induced bone loss through comparing femoral gene expression profiles in HFD-fed mice $v$. normal diet-fed mice during the growth stage and have shown that genes associated with bone resorption are increased and bone formation is decreased in mice fed a $\mathrm{HFD}^{(14)}$. Our earlier studies have shown that there are differences in peak bone mass (PBM) acquisition in young female and male adults. Difference in bone mineral density in males and females could be attributed to an increase in bone size in males during growth. However, how PBM is influenced in the presence of a HFD in both males and females remains uninvestigated.

In the present study we evaluated the bone architecture of the femur and proximal and mid-shaft tibia by microcomputed tomography in mice fed a HFD for 10 weeks to determine whether a HFD influences bone structure in males and females differentially. Further, to determine the effect of a HFD on the functions of osteoblasts, we cultured bone marrow stromal/osteoblastic cells (BMSC) for up to $21 \mathrm{~d}$. The areas of positive colony-forming units at day 14 and $\mathrm{Ca}$ nodules at day 21 were measured in addition to the expression of adipogenic, osteogenic and osteoclastogenic genes.

\section{Materials and methods}

\section{Animals and diets}

All animal care and experimental procedures were approved by the Institutional Animal Ethics Committee (IAEC) following the guidelines of the Committee for the Purpose of Control and Supervision of Experiments on Animals and conform to the international norms of the Indian National Science Academy.
Male and female C57BL/6 mice ( $n$ 24; aged 4 weeks), obtained from the National Laboratory Animal Centre at the Council of Scientific and Industrial Research - Central Drug Research Institute, Lucknow, India, were used and received humane care in compliance with the Guidelines for the Care and Use of Laboratory Animals, and were randomly assigned to four groups of eight mice each. Animals were kept in a $12 \mathrm{~h}$ light $-12 \mathrm{~h}$ dark cycle, with controlled temperature $\left(22-24^{\circ} \mathrm{C}\right)$ and humidity (50-60\%) and were given ad libitum access to water and food. The control group of female and male mice was fed with a standard chow diet and the second group was fed with a HFD (D12492, 60\% energy from fat; Research Diets, Inc.) (see online supplementary Tables S1 and S2) that was given by weight in grams for 10 weeks, respectively. Body weight was recorded weekly.

\section{Biochemical analysis}

At the end of 10 weeks of study, mice were euthanised and autopsied. Blood was collected in tubes containing EDTA and plasma was immediately separated by centrifugation and frozen at $-80^{\circ} \mathrm{C}$. Total cholesterol, TAG, HDL and LDL were determined by colorimetric assay in a semi-automatic biochemistry analyser ${ }^{(15)}$. On the basis of our previously published protocols, plasma osteocalcin (OCN) (midportion) and carboxy-terminal collagen crosslinks (CTx) levels were determined with ELISA kits (Immunodiagnostic Systems Ltd) following the manufacturer's protocols ${ }^{(16,17)}$.

\section{Mineralisation of bone marrow cells}

At the end of 10 weeks, bone marrow cells were isolated from mice and cultured according to a previously published protocol $^{(16,18)}$. Briefly, the femora were excised aseptically, cleaned of soft tissues, and washed three times, $15 \mathrm{~min}$ each, in a culture medium. The epiphyses of femora were cut off and the marrow was flushed out in $20 \mathrm{ml}$ of minimum essential medium ( $\alpha$-MEM). Released bone marrow cells were collected and plated $\left(2 \times 10^{6}\right.$ cells/well $)$ in twelve-well plates in the culture medium, consisting of $\alpha$-MEM, supplemented with $10 \%$ fetal bovine serum, 100 nm-dexamethasone, $10 \mathrm{~mm}-\beta$-glycerophosphate and $50 \mu \mathrm{g} / \mathrm{ml}$ ascorbic acid. Cells were cultured for $21 \mathrm{~d}$ at $37^{\circ} \mathrm{C}$ in a humidified atmosphere of $5 \% \mathrm{CO}_{2}$ and $95 \%$ air, and the medium was changed every $48 \mathrm{~h}$. At day 14 of culture, cells were washed twice with PBS, then plates were fixed by keeping them at $-70^{\circ} \mathrm{C}$ for $1 \mathrm{~h}$, and then brought to room temperature to determine alkaline phosphatase (ALP) activity. The rate of the reaction here is directly proportional to the enzyme activity, which itself is proportional to osteoblast differentiation. Optical density was measured at $405 \mathrm{~nm}$ with a microplate reader ${ }^{(19)}$. After $21 \mathrm{~d}$, the differentiated cells were fixed in $4 \%$ formaldehyde for $20 \mathrm{~min}$ at room temperature and rinsed once in PBS. After fixation, the specimens were processed for staining with $40 \mathrm{~mm}$-Alizarin Red-S, which stains areas rich in nascent $\mathrm{Ca}$. The stain was extracted and quantified by the ELISA plate reader at $405 \mathrm{~nm}^{(16,19-21)}$ 


\section{Induction of adipogenic differentiation in bone marrow cells}

For adipogenic differentiation, $1 \times 10^{7}$ bone marrow cells from all groups were seeded in twenty-four-well plates and cultured in an adipogenic medium (Dulbecco's modified Eagle's medium) containing $1.0 \mu \mathrm{m}$-dexamethasone, $0.5 \mathrm{~mm}$ isobutylmethylxanthine, $100 \mu \mathrm{m}$-indomethacin, 10\% FBS and insulin $(10 \mu \mathrm{g} / \mathrm{ml})$, for $7 \mathrm{~d}$. On day 3, medium was replaced with complete growth medium containing only insulin $(10 \mu \mathrm{g} / \mathrm{ml})$. Medium was replaced every third day and continued for $21 \mathrm{~d}$. Cells were fixed after $21 \mathrm{~d}$ in $4 \%$ paraformaldehyde and stained with Oil Red O. To quantify the incorporation of lipid, the area stained with Oil Red $\mathrm{O}$ was measured by taking photomicrographs ${ }^{(15,19,22,23)}$. For the extraction of the Oil Red O stain, $500 \mu \mathrm{l}$ of $70 \%$ propanol were added to the stained cells and left at $37^{\circ} \mathrm{C}$ for $20 \mathrm{~min}$. The extracted stain was quantified by the ELISA plate reader at $490 \mathrm{~nm}^{(19,22)}$.

\section{Glucose tolerance test}

At the end of 10 weeks of treatment with the HFD, mice were fasted overnight and blood glucose was measured from the tail vein at 0 (before intraperitoneal injection), 15, 30, 60, 90 and $120 \mathrm{~min}$ (after intraperitoneal injection) (injection of $50 \%$ D-glucose (dextrose) at $3 \mathrm{ml} / \mathrm{kg}$ body weight). Blood glucose measurements were done using an Optimum Exceed glucometer (Abbott Diabetes Care Inc.). AUC for glucose tolerance was compared among different groups ${ }^{(24)}$

\section{Real-time PCR for quantification of the messenger RNA levels of various bone-specific genes}

From frozen left femora, total RNA was extracted with TRIzol (Invitrogen) according to the manufacturer's instructions. The concentration and purity of RNA were determined by measuring the absorbance at $260 \mathrm{~nm}$ (A260) and the ratio of the absorbance at $260 \mathrm{~nm}$ to the absorbance at $280 \mathrm{~nm}$ (A260/A280), respectively. Primers were designed using the Universal Probe Library (Roche Applied Sciences) for genes, as presented in Table 1. Complementary DNA was synthesised with the Revert Aid cDNA Synthesis Kit (Fermentas) using $2 \cdot 0 \mu \mathrm{g}$ of total RNA. SYBR Green chemistry was used to perform a quantitative determination of the relative expression of transcripts for all genes. Relative mRNA expression of all the genes were analysed using the Light Cycler 480 (Roche Molecular Biochemicals) real-time PCR machine, as we have reported previously. Table 1 shows the primer sequences of various murine genes used for quantitative PCR. The reaction volume was $20 \mu \mathrm{l} /$ well on ninety-six-well plates. Transcript levels were normalised to glyceraldehyde-3-phosphate dehydrogenase levels. The relative messenger RNA (mRNA) expression levels were calculated according to the comparative cycle threshold (CT) $\left({ }^{\Delta \Delta C T}\right)$ method, as described by the manufacturer (LightCycler ${ }^{\circledR} 480$ Instrument, Software Version 1.5, February 2008; http://www.roche-applied-science.com). Target quantity is normalised to endogenous control and relative to a calibrator and is calculated using the formula: target amount $2^{-\Delta \Delta \mathrm{CT}(16,21,25)}$.
Table 1. Quantitative PCR primers

\begin{tabular}{|c|c|c|}
\hline Gene & Primer sequence & $\begin{array}{l}\text { Accession } \\
\text { number }\end{array}$ \\
\hline \multirow[t]{2}{*}{ OCN } & F: TGAGGACCATCTTCTGCTCA & NM_001032298 \\
\hline & R: TGGACATGAAGGCTTTGTCA & \\
\hline \multirow[t]{2}{*}{ Col-1 } & F: CATGTTCAGCTTTGTGGACCT & NM_007742.3 \\
\hline & R: GCAGCTGACTTCAGGGATGT & \\
\hline \multirow[t]{2}{*}{$R U N X-2$} & F: CCCGGGAACCAAGAAATC & AF053956.1 \\
\hline & R: CAGATAGGAGGGGTAAGACTGG & \\
\hline \multirow[t]{2}{*}{$P P A R-\gamma$} & F: GAAAGACAACGGACAAATCACC & NM_011146.3 \\
\hline & R: GGGGGTGATATGTTTGAACTTG & \\
\hline \multirow[t]{2}{*}{$a P-2(F A B P 4)$} & F: GAAAACGAGATGGTGACAAGC & NM_024406.2 \\
\hline & R: GCCCTTTCATAAACTCTTGTGG & \\
\hline \multirow[t]{2}{*}{$O P G$} & F: AGCCATTGCACACCTCAC & NM_011613 \\
\hline & R: CGTGGTACCAAGAGGACAGAGT & \\
\hline \multirow[t]{2}{*}{ RANKL } & F: AGCCATTTGCACACCTCAC & NM_011613 \\
\hline & R: CGTGGTACCAAGAGGACAGAGT & \\
\hline \multirow[t]{2}{*}{ TRAP } & F: CGTCTCTGCACAGATTGCAT & NM_001102405.1 \\
\hline & R: AAGCGCAAACGGTAGTAAGG & \\
\hline \multirow[t]{2}{*}{ RANK } & F: AGAGGCATTATGAGCATCTCG & BC019185.1 \\
\hline & R: CGAGTGCACTTAGAGGACAGGT & \\
\hline \multirow[t]{2}{*}{ GAPDH } & F: AGCTTGTCATCAACGGGAAG & DQ403054.1 \\
\hline & R: TTTGATGTTAGTGGGGTTCTCG & \\
\hline
\end{tabular}

$O C N$, osteocalcin; F, forward; $\mathrm{R}$, reverse; Col-1, collagen type-1; RUNX-2, runtrelated transcription factor $2 ; a P-2$, adipocyte protein $2 ; F A B P 4$, fatty acid binding protein 4; OPG, osteoprotegerin; RANKL, receptor activator of NF-kB ligand; $T R A P$, tartrate-resistant acid phosphatase; RANK, receptor activator of $\mathrm{NF}-\mathrm{kB}$; $G A P D H$, glyceraldehyde-3-phosphate dehydrogenase.

\section{Microcomputed tomography}

Microcomputed tomography of excised bones was carried out using the SkyScan 1076 micro-computed tomography $(\mu \mathrm{CT})$ scanner (Bruker microCT) as described before ${ }^{(21,26)}$. The bone samples were scanned at a resolution of $9 \mathrm{~mm}$. Reconstruction was carried out using SkyScan Nrecon software (Bruker microCT). The X-ray source was set at $50 \mathrm{kV}$ and $200 \mathrm{~mA}$, with a pixel size of $9 \mathrm{~mm}$. A total of 100 projections were acquired over an angular range of $180^{\circ}$. The trabecular bone was selected by drawing ellipsoid contours with the computed tomography analyser (CTAn) software (SkyScan; Bruker microCT). Trabecular bone volume/tissue volume (BV/TV), trabecular number (Tb.N) and trabecular separation (Tb.Sp) of the distal femoral metaphysis (covering secondary spongiosa and the secondary ossification centre) and proximal tibial metaphysis were calculated by the mean intercept length method. Three-dimensional parameters were based on analysis of a Marching cubes-type model with a rendered surface. CTVol software (Skyscan) was used to create a three-dimensional model of the bones ${ }^{(20,21)}$. Lengths of femora were measured. After that femora were subjected to the three-point bending test with the bone strength tester model TK 252C (Muromachi Kikai Co. Ltd) ${ }^{(19)}$.

\section{Statistical analysis}

Values were expressed as mean values with their standard errors. Comparisons of each parameter among all of the groups were analysed via one-way ANOVA followed by Neuman-Keuls post hoc tests using Prism version 3.0 software (GraphPad). Probability values of $P<0.05$ were considered to be statistically significant. In addition, two-way ANOVA was 
used to determine the effects of diet and sex in all groups, using Prism version 3.0 software.

\section{Results}

\section{Differential susceptibility to obesity between male and female mice}

The HFD was used to induce the metabolic syndrome and the stage of disorder was defined by taking body weight at each point for the four groups as shown in Fig. 1. Starting from 4 weeks of age, the body weight of animals fed the HFD was significantly greater than that of mice fed the control diet $(P<0 \cdot 05)$. From this time on, the difference between all the groups increased consistently. At the end of the 10-week study on experimental diets, female mice on the HFD weighed about $27 \%$ heavier than female mice on control chow diet (18.5 (sE 0.2) and 20.8 (SE 0.9) g for the control and HFD female mice, respectively), while male mice on the HFD weighed about $15.8 \%$ heavier than male mice on the chow diet (22.5 (sE 0.6) and 24.9 (sE 0.8) g for control and HFD male mice, respectively). However, data suggest that the susceptibility to weight gain as percentage weight gain was higher in male mice compared with female mice. Comparison of data within the control diets (chow) at the end of 10-week study shows that male mice showed a $28 \%$ increase in body weight while female mice showed a $9 \cdot 1 \%$ increase. The results of two-way ANOVA indicated that both $\operatorname{diet}(P=0.029)$ and sex $(P<0 \cdot 0001)$ individually affected body weight, but no interaction was found between these effects.

\section{Effect of high-fat diet on lipid profile and development of} hyperinsulinaemia in males

Table 2 shows changes in serum lipid parameters for the four groups. Circulating levels of total cholesterol were increased

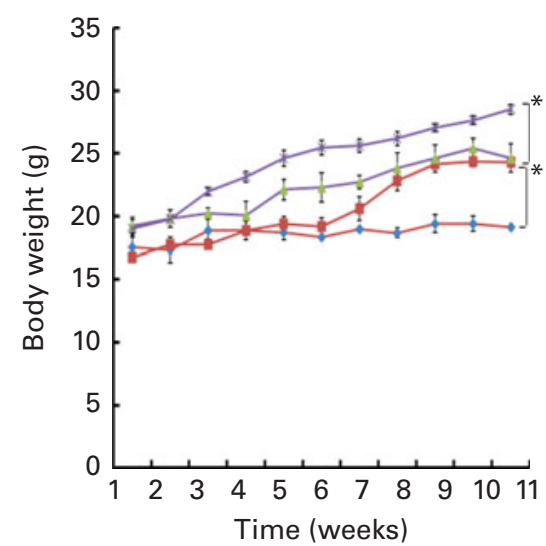

Fig. 1. High-fat diet (HFD) feeding induces differential susceptibility to obesity in male and female mice. Feeding the HFD for 10 weeks to C57BL/6 mice increased body weights in both males and females but the weight gain in male mice showed more increase as compared with the females. Values are means for eight mice per group, with standard errors represented by vertical bars. * Mean values were significantly different compared with the HFD group $(P<0.05)$. Two-way ANOVA was performed to assess the effects of diet $(P=0.029)$, sex $(P<0.0001)$ and of their interaction (diet $\times$ sex; NS) on body weights. $\rightarrow$, Chow-fed females;,-- HFD-fed females; $\rightarrow$, chow-fed males; $*$, HFD-fed males. in both female and male HFD-fed mice; however, male mice on the HFD had about $20-25 \%$ higher serum cholesterol concentrations compared with female mice fed the HFD. HDL, considered to be the good cholesterol, is actually a carrier molecule that transports cholesterol in the blood. Levels were significantly less (about 60\%) in male HFD mice compared with those fed the chow diet; however, 10 weeks of HFD treatment did not affect the females. LDL is a carrier molecule that transports cholesterol and TAG in the blood from the liver to the body's cells and a high level of LDL is associated with a high risk of heart attack as it indicates arteryclogging cholesterol in the blood. Data show that LDL levels were significantly $(P<0.05)$ higher in male HFD-fed mice compared with female HFD-fed mice. TAG are the principal fat in the diet and the serum levels of these indicate how much fat is moving through the body, potentially to clog the arteries. Data show that TAG levels were high (about $70 \%$ ) in male HFD-fed animals as compared with the chow dietfed male mice. Comparison of data with the female group shows that male mice had the susceptibility towards higher TAG levels $(P<0 \cdot 01)$. However, TAG levels of female chow $v$. HFD animals remain unchanged. The results of two-way ANOVA indicated that diet was the main factor to increase total cholesterol $(P=0.0002)$, TAG $(P=0.0092)$ and LDL $(P=0.0036)$ levels. However, for HDL sex seemed to be a greater contributor in decreasing the HDL levels $(P=0 \cdot 0011)$. Assessment of plasma glucose concentrations in males and females shows that glucose levels increased in HFD female mice over time compared with mice on the control chow diet. However, glucose levels in HFD male mice remained constant $v$. the control chow diet animals. After 10 weeks of HFD treatment, glucose tolerance was impaired in female mice as compared with age-matched male mice (Fig. 2).

\section{Effect of high-fat diet on trabecular bone architecture as shown by microcomputed tomography}

Having checked the status of the lipid profile after 10 weeks of HFD treatment, we next studied the effect of the HFD on bone. Tables 3 and 4 show gross observations of the trabecular architecture by three-dimensional microcomputed tomography of the distal femur and tibia in both male and female groups. Deterioration in the trabecular structures is clearly visible in HFD groups (Fig. 3(a)). Data show that 10 weeks of HFD treatment to both males and females resulted in significant decreases in BV/TV and Tb.N, when compared with the chow group (control group). Comparison of data between male and female groups shows that loss of percentage bone was the same in both the sexes. However, Tb.Sp increased in both the males and females but a greater increase was observed in males on the HFD than female mice fed the HFD. Likewise, the structural model index (SMI) was used to assess plate-like trabeculae. A higher SMI as attained by 10 weeks of HFD treatment indicates deterioration of trabeculae from a plate- to a rod-like structure that was significantly more in the male group as compared with the females. As trabecular micro-architecture is an independent determinant of fractures we therefore also assessed 


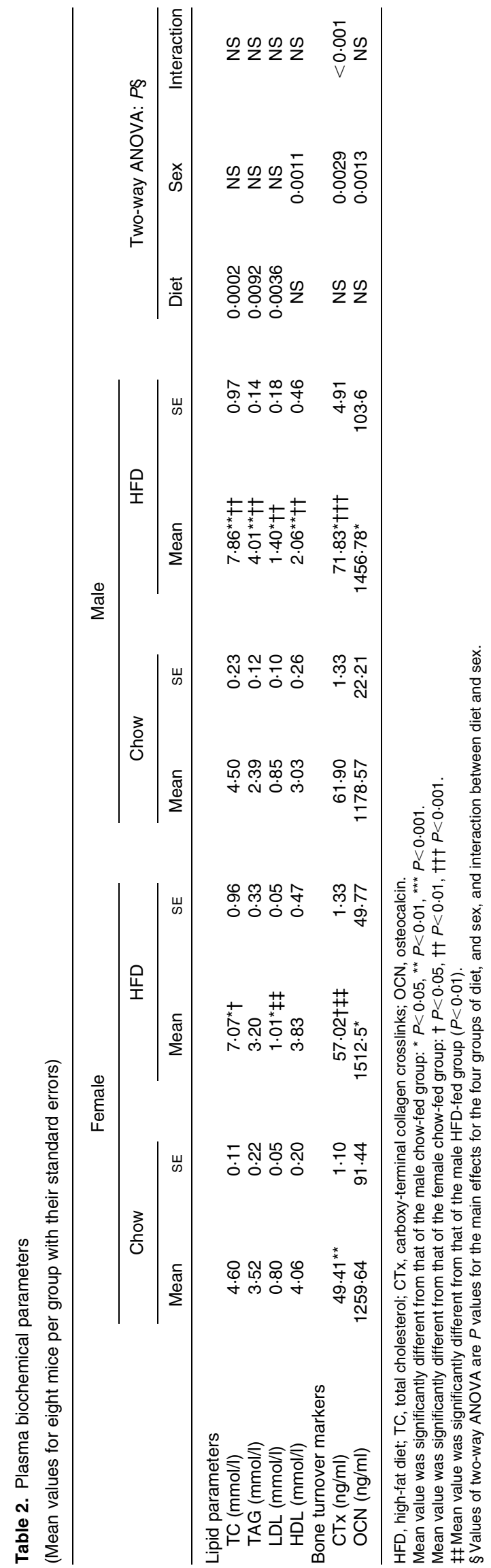

micro-architecture at the cortical sites. The HFD with $60 \%$ energy as fat for 10 weeks did not produce any change in cortical thickness in either the male or female animals (Table 3, Fig. 3(b)), when compared with their chow-fed counterparts; this was corroborated by no changes in the energy to failure and maximum stiffness in males fed the HFD and no change in the energy to failure in females fed with the HFD (Table 5). To assess whether the HFD affects the growth of animals, as these were young growing animals, we measured femoral length at the end of the experiment. We observed no change in the growth (length) of the femur as compared with the control chow diet-fed animals, both males and females (Table 3). The results of two-way ANOVA indicated that in the case of femoral metaphysis, diet had a significant effect on BV/TV $(P<0.0001)$, Tb.Sp $(P=0.0024)$, Tb.N $(P<0.0001)$ and SMI $(P<0.0001)$; however, in the case of Tb.N $(P=0.0175)$ and SMI $(P<0.0001)$, sex was also a factor that affected the results. There was no significant interaction between diet and sex on either of the variables. Similarly, in the tibial metaphysis region diet had a significant effect $(P<0.0001)$ on BV/TV, Tb.Sp, Tb.N and SMI. A significant interaction between diet and sex affected Tb.Sp in tibia $(P=0.0078)$, where males exhibited greater Tb.Sp as compared with the females.

\section{Effects of high-fat diet on bone turnover markers in males and females}

The established bone formation marker, plasma OCN, and a resorption marker (CTx) were studied to corroborate our findings on bone deterioration as observed by us. Plasma CTx levels were higher in the HFD-treated mice compared with the control chow groups. Data suggest that the HFD impairs bone loss via increased osteoclastic bone resorption. Although we found higher levels of plasma CTx in both HFD-fed male and female mice $(P<0.05)$, collagen loss was significantly more in the male group $(P<0.01)$ as compared with the females (Table 2). Results of two-way ANOVA indicated that both diet $(P=0.0029)$ and sex $(P<0.0001)$ had significant

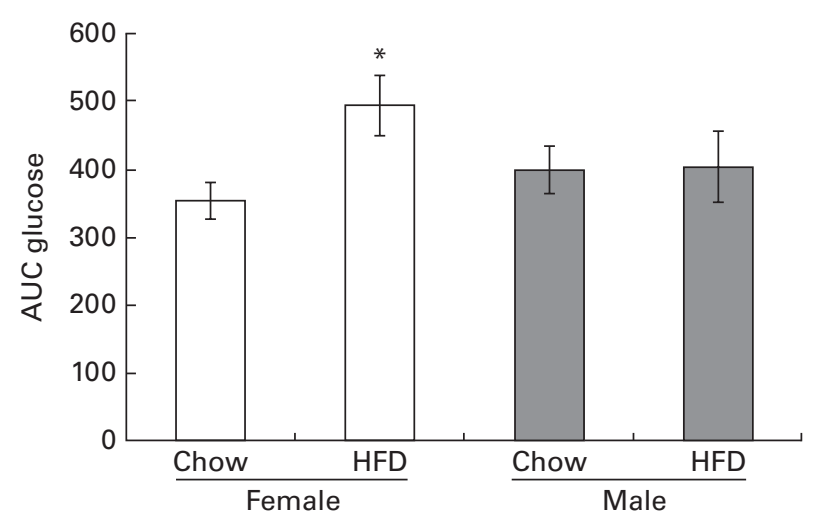

Fig. 2. Effect of oral glucose tolerance test in female and male chow-fed and high-fat diet (HFD)-fed mice. Values are means for eight mice per group, with standard errors represented by vertical bars. * Mean value was significantly different from that of the female chow-fed group $(P<0.05)$. Two-way ANOVA was performed to assess the effects of diet (NS), sex (NS) and of their interaction (diet $\times$ sex; NS) on glucose tolerance. 
Table 3. Micro-architectural parameters of femoral metaphyseal region

(Mean values for eight mice per group with their standard errors)

\begin{tabular}{|c|c|c|c|c|c|c|c|c|c|c|c|}
\hline & \multicolumn{4}{|c|}{ Female } & \multicolumn{4}{|c|}{ Male } & & & \\
\hline & \multicolumn{2}{|c|}{ Chow } & \multicolumn{2}{|l|}{ HFD } & \multicolumn{2}{|c|}{ Chow } & \multicolumn{2}{|c|}{ HFD } & \multicolumn{3}{|c|}{ Two-way ANOVA: P§ } \\
\hline & Mean & SE & Mean & SE & Mean & SE & Mean & SE & Diet & Sex & Interaction \\
\hline \multicolumn{12}{|c|}{$\begin{array}{l}\text { Trabecular parameter } \\
\text { of femoral metaphysis }\end{array}$} \\
\hline BV/TV (\%) & $5 \cdot 81$ & 0.54 & $1.78^{* \star *}+\dagger \dagger$ & 0.66 & 7.06 & 0.77 & $3.07^{\star \star \star}+\dagger \dagger$ & 0.68 & $<0.0001$ & NS & NS \\
\hline Tb.Sp (mm) & 0.42 & 0.02 & $0.47 \ddagger$ & 0.02 & 0.42 & 0.03 & $0.56^{\star \star}+\dagger$ & 0.04 & 0.0024 & NS & NS \\
\hline Tb.N (/mm) & $0.78^{*}$ & 0.06 & $0.28^{* \star *} \dagger \dagger$ & 0.09 & 1.08 & $0 \cdot 12$ & $0.44^{\star \star \star} \dagger$ & 0.08 & $<0.0001$ & 0.0175 & NS \\
\hline SMI & $2 \cdot 12^{\star \star \star}$ & 0.09 & $2 \cdot 43 † \dagger \neq \ddagger$ & 0.03 & $2 \cdot 41$ & 0.03 & $2 \cdot 7^{\star *}+\dagger \dagger$ & 0.03 & $<0.0001$ & $<0.0001$ & NS \\
\hline \multicolumn{12}{|c|}{$\begin{array}{l}\text { Cortical parameters } \\
\text { of femora }\end{array}$} \\
\hline Cs.Th (mm) & 0.09 & 0.005 & $0.853^{\star \star \star} \ddagger \ddagger \ddagger$ & 0.001 & 0.13 & 0.001 & $0.14 \dagger+\dagger$ & 0.002 & NS & $<0.0001$ & NS \\
\hline \multicolumn{12}{|l|}{ Femoral length } \\
\hline Length $(\mathrm{cm})$ & $1 \cdot 46^{*}$ & 0.035 & 1.55 & 0.006 & 1.59 & 0.025 & 1.50 & 0.049 & NS & NS & 0.009 \\
\hline
\end{tabular}

HFD, high-fat diet; BV/TV, bone volume/tissue volume; Tb.Sp, trabecular separation; Tb.N, trabecular number; SMI, structure model index; Cs. Th, cortical thickness.

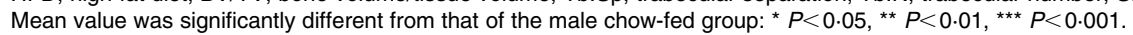

Mean value was significantly different from that of the female chow-fed group: $\dagger P<0.05, \dagger \dagger P<0.01, \dagger \dagger \dagger P<0.001$

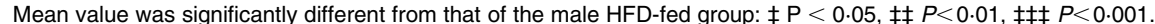

$\S$ Values of two-way ANOVA are $P$ values for the main effects for the four groups of diet, and sex, and interaction between diet and sex.

effects on CTx levels, with higher bone resorption in males as compared with females.

OCN levels that are indicative of bone formation as compared with resorption were assayed in the plasma and data suggest that whereas females could not compensate for the bone loss, the male group had modestly increased OCN levels (Table 2). Similarly, two-way ANOVA results showed that sex contributed to significant changes in OCN levels $(P=0.0013)$, with higher OCN levels in males.

\section{Effect of high-fat diet on modulation of progenitor cells in the bone marrow}

To determine the effect of dietary fat on bone marrow stromal cells, BMSC from long bone were isolated and cultured at the end of the 10-week HFD treatment. BMSC were differentiated into osteoblasts and adipocytes, as both cell types differentiate from mesenchymal cells and share a reciprocal relationship with each other. Fig. 4(a) shows that BMSC following from HFD treatment had significantly reduced ALP (osteoblast proliferation) activity in both males and females (about 50-60\%) in response to osteogenic stimulus as compared with chow-fed (control) animals. Comparison of data between males and females shows that there was no significant difference in the decrease in ALP, as both the groups showed an equal response towards the decrease. Two-way ANOVA results corroborated the above findings suggesting the effect of both diet $(P<0.0001)$ and sex $(P=0.0008)$ on ALP activity.

Further we analysed the mineralising ability of the BMSC that started to form mineralising nodules at day 14 of culture and maximal $\mathrm{Ca}$ nodules were observed at day 21 of culture. At day 21 of culture, the number of Alizarin Red-stained Ca nodules (Fig. 4(b)) was decreased about 30\% $(P<0.05)$ in mice fed the HFD compared with control chow diet-fed mice. Extraction and quantification of the dye showed that HFD treatment in males as compared with females rendered significantly less $(P<0.01)$ mineralisation (Fig. 4(c)), suggesting the effect of both diet $(P=0.0002)$ and sex $(P=0.0003)$ on the mineralisation of bone marrow cells.

Table 4. Micro-architectural parameters of tibial metaphyseal region

(Mean values for eight mice per group with their standard errors)

\begin{tabular}{|c|c|c|c|c|c|c|c|c|c|c|c|}
\hline & \multicolumn{4}{|c|}{ Female } & \multicolumn{4}{|c|}{ Male } & & & \\
\hline & \multicolumn{2}{|c|}{ Chow } & \multicolumn{2}{|c|}{ HFD } & \multicolumn{2}{|c|}{ Chow } & \multicolumn{2}{|c|}{ HFD } & \multicolumn{3}{|c|}{ Two-way ANOVA: P§ } \\
\hline & Mean & SE & Mean & SE & Mean & SE & Mean & SE & Diet & Sex & Interaction \\
\hline \multicolumn{12}{|c|}{ Trabecular parameters of tibial metaphysis } \\
\hline BV/TV (\%) & 3.37 & 0.14 & $1 \cdot 37^{\star \star \star}+\dagger$ & 0.09 & 4.41 & 0.72 & $1.43^{\star \star \star}+\dagger$ & 0.17 & $<0.0001$ & NS & NS \\
\hline Tb.Sp (mm) & 0.54 & 0.01 & $0.65^{\star \star \star} \dagger \ddagger$ & 0.04 & 0.46 & 0.03 & $0 \cdot 75^{\star \star \star}+\dagger †$ & 0.03 & $<0.0001$ & NS & 0.0078 \\
\hline Tb.N (/mm) & $0.53^{*}$ & 0.01 & $0.23^{\star * *}+\dagger$ & 0.02 & 0.75 & $0 \cdot 11$ & $0.23^{\star \star \star}+\dagger \dagger$ & 0.02 & $<0.0001$ & NS & NS \\
\hline SMI & $2 \cdot 39^{\star *}$ & 0.07 & $2.58+\dagger$ & 0.03 & $2 \cdot 33$ & 0.03 & $2 \cdot 64^{\star \star \star}+\dagger$ & 0.02 & $<0.0001$ & NS & NS \\
\hline
\end{tabular}

HFD, high-fat diet; BV/TV, bone volume/tissue volume; Tb.Sp, trabecular separation; Tb.N, trabecular number; SMI, structure model index.

Mean value was significantly different from that of the male chow-fed group: ${ }^{\star} P<0.05,{ }^{\star *} P<0.01,{ }^{\star \star *} P<0.001$.

Mean value was significantly different from that of the female chow-fed group: $\dagger P<0.05, \dagger \dagger P<0.01, \dagger \dagger \dagger P<0.001$.

$\ddagger$ Mean value was significantly different from that of the male HFD-fed group $(P<0.05)$.

$\S$ Values of two-way ANOVA are $P$ values for the main effects for the four groups of diet, and sex, and interaction between diet and sex. 
(a)

Male

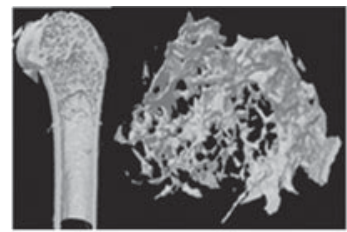

Female
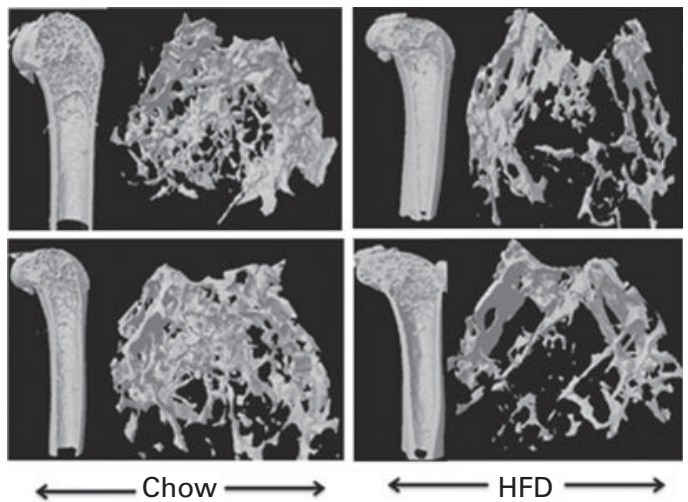

(b)

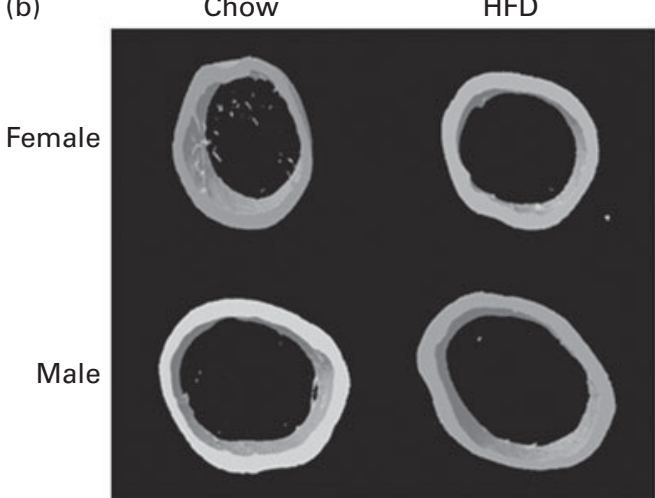

Fig. 3. High-fat diet (HFD) feeding induced differential effects in trabecular bone micro-architecture of femoral and tibial metaphysis regions by microcomputed tomography. (a) Representative microcomputed tomography images (longitudinal and transverse cross-sectional three-dimensional view) of the distal femoral metaphysis of male and female chow- and HFD-fed mice by microcomputed tomography. (b) Representative microcomputed tomography images of the cross-sectional three-dimensional view of the femoral diaphyseal region of female and male chow- and HFD-fed mice. HFD treatment did not induce changes in cortical thickness.

Differentiation of BMSC into adipocytes as in Fig. 4(d) shows that BMSC obtained from HFD-fed mice (both male and female) favoured the progression towards adipogenic lineage and showed more accumulation of lipid droplets. Fig. 4(d) shows adipocytes stained with Oil Red O from the four groups. After $21 \mathrm{~d}$ of differentiation, absorbance data of extracted Oil Red $O$ from the cells showed that there was increased adipogenesis in male and female HFD-fed mice compared with chow-fed mice; however, we found increased adipogenesis in male HFD-fed mice compared with female HFD-fed mice $(P<0.001)$ (Fig. $4(\mathrm{e}))$ as confirmed by twoway ANOVA $(P<0.0001$ for diet and $P<0.0001$ for sex).

\section{Direct effect of high-fat diet at messenger RNA level on femur long bone}

We next assessed the status of osteoblasts, osteoclasts and adipocytes by quantifying mRNA levels of various genes after HFD treatment. Table 6 shows the mRNA levels of adipogenic, osteogenic and osteoclast specific genes. For osteoblasts we assessed runt-related transcription factor 2 (RUNX-2), type I collagen (Col-1) and OCN. Two-way ANOVA analysis suggests that diet was the main factor to affect Col-1 $(P=0.0022)$ and OCN $(P=0.0002)$ levels. Realtime PCR shows that mRNA levels remained unchanged in the females fed the HFD for RUNX- 2 and Col- 1 but were significantly reduced for OCN levels. In males the reduction in osteoblastic gene expression was more pronounced when compared with the female group fed the HFD for 10 weeks. Since osteoblasts and adipocytes derive from the same mesenchymal stem cells, our data for adipogenic genes show that adipocytic transcription factors were significantly more (PPAR $\gamma$ 2-fold and adipocyte protein-2 (aP-2) 6-fold) as compared with not only the control chow diet-fed animals but also with the females fed the HFD. Two-way ANOVA analysis confirms that diet and sex individually affected the levels of PPAR $y$ and aP-2 $(P<0 \cdot 0001)$, with a significant interaction also between diet and sex $(P=0.0014$ for PPAR $\gamma$ and $P<0.0001$ for aP-2). Data in osteoclasts for the resorptive genes robustly increased tartrate-resistant acid phosphatase (TRAP) about 3-fold and receptor activator of NF-кB (RANK) about 20-fold in the males on the HFD for 10 weeks whereas this expression remained unchanged in the female counterparts when compared with their controls. Two-way ANOVA for diet and sex shows $P=0.0013$ for TRAP and $P<0.0001$ for RANK expression. However, interaction between diet and sex was also found to affect mRNA levels of TRAP $(P=0 \cdot 0013)$ and RANK $(P<0 \cdot 0001)$. During bone remodelling, osteoblasts produce important paracrine signals that promote osteoclastogenesis. Osteoprotegerin (OPG) and receptor activator of NF- $\mathrm{KB}$ ligand (RANKL) produced by osteoblasts

Table 5. Biomechanical strength of femora

(Mean values for eight mice per group with their standard errors)

\begin{tabular}{|c|c|c|c|c|c|c|c|c|c|c|c|}
\hline & \multicolumn{4}{|c|}{ Female } & \multicolumn{4}{|c|}{ Male } & & & \\
\hline & \multicolumn{2}{|c|}{ Chow } & \multicolumn{2}{|c|}{ HFD } & \multicolumn{2}{|c|}{ Chow } & \multicolumn{2}{|c|}{ HFD } & \multicolumn{3}{|c|}{ Two-way ANOVA: $P \S$} \\
\hline & Mean & SE & Mean & SE & Mean & SE & Mean & SE & Diet & Sex & Interaction \\
\hline Power (N) & 14.50 & $2 \cdot 46$ & $17 \cdot 83$ & $2 \cdot 90$ & 11.37 & 1.43 & $14 \cdot 20$ & 1.65 & NS & NS & NS \\
\hline Stiffness (N/mm) & $41 \cdot 80$ & 3.42 & $62 \cdot 45^{\star} \dagger \ddagger$ & 9.09 & 33.13 & $7 \cdot 10$ & 33.60 & $5 \cdot 80$ & NS & 0.0092 & NS \\
\hline Energy (mJ) & 14.40 & 0.70 & $18 \cdot 20$ & 0.78 & 15.46 & 1.94 & $25 \cdot 30$ & 3.48 & NS & NS & NS \\
\hline
\end{tabular}

HFD, high-fat diet.

* Mean value was significantly different from that of the male chow-fed group $(P<0.05)$.

$\dagger$ Mean value was significantly different from that of the female chow-fed group $(P<0.05)$

$\ddagger$ Mean value was significantly different from that of the male HFD-fed group $(P<0.05)$.

$\S$ Values of two-way ANOVA are $P$ values for the main effects for the four groups of diet, and sex, and interaction between diet and sex. 
(a)

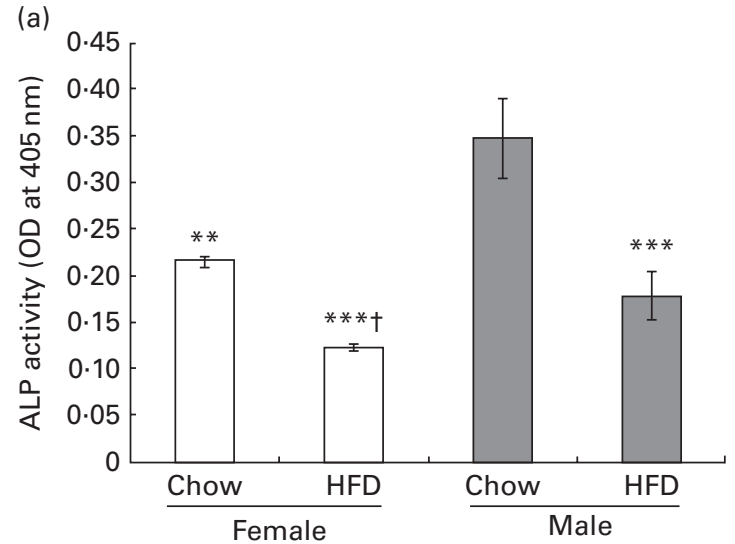

(b)

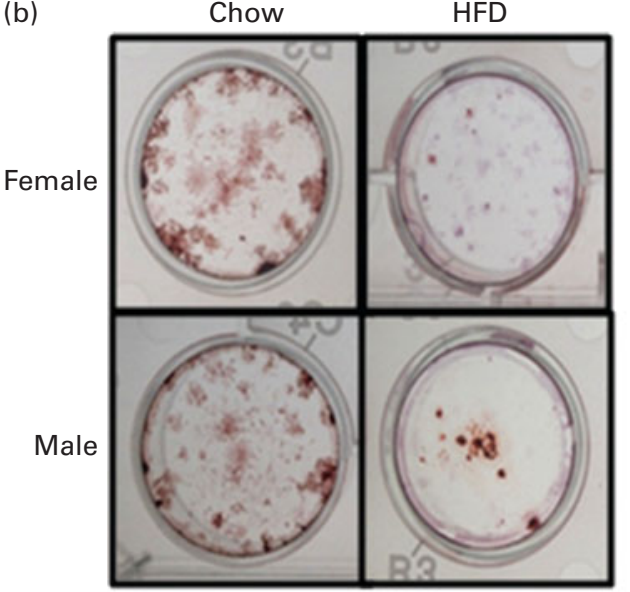

(d)

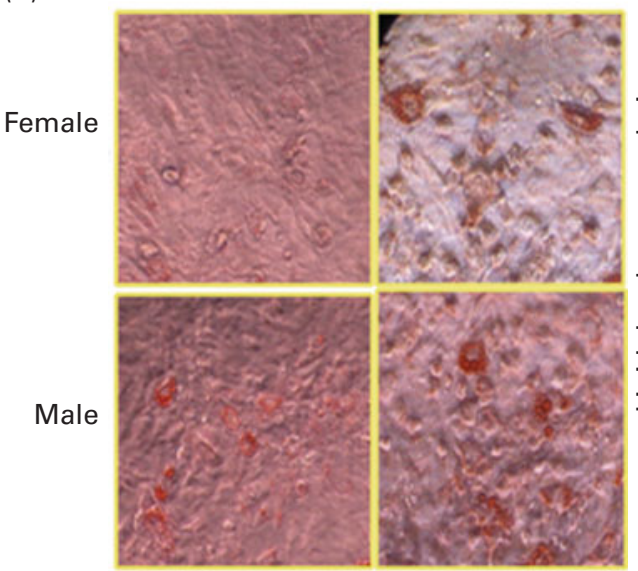

(c)

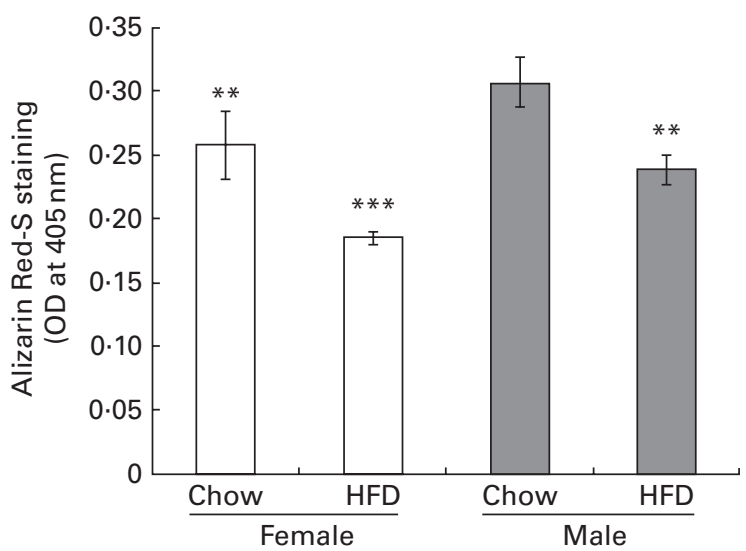

(e)

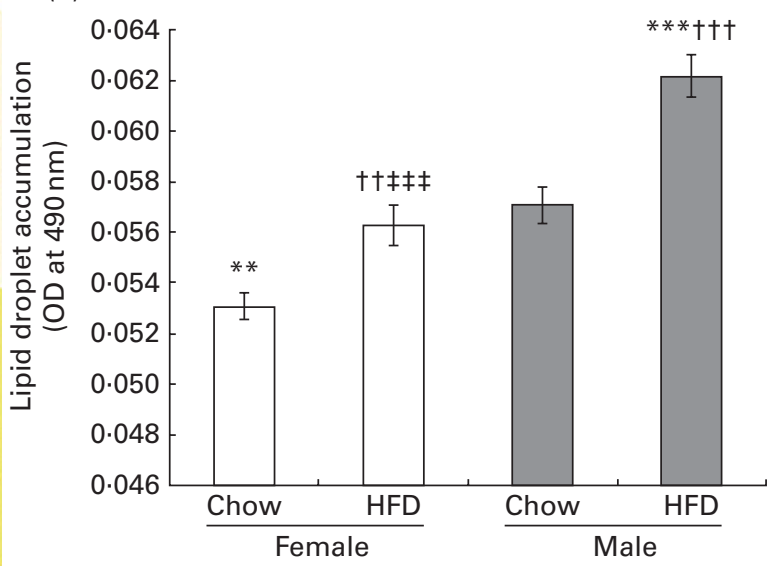

Fig. 4. Modulation of osteoprogenitor cells in the bone marrow by high-fat diet (HFD) feeding. For the alkaline phosphatase (ALP) assay, $1 \times 10^{5}$ cells were plated in forty-eight-well plates; for the mineralisation assay $2 \times 10^{6}$ bone marrow cells (BMC)/well were plated onto twelve-well plates. (a) ALP activity. (b) Calcium deposition by osteoblasts was determined by Alizarin Red-S staining of the cultures at the end of the experiment for all four groups. Representative photomicrographs show mineralisation nodules for the four groups stained with Alizarin-S dye. (c) For quantification Alizarin-S dye was extracted and mineralisation quantified. (d) To assess the differentiation of BMC to adipocytes, $1 \times 10^{7} \mathrm{BMC} /$ well were differentiated into adipocytes of four groups. Cultures were maintained for $21 \mathrm{~d}$. At the end of the experiment, cells were stained with Oil Red O. Photomicrographs show that HFD feeding inhibits the differentiation of BMC to adipocytes. (e) Quantification of lipid droplet accumulation in the cells by extraction of the dye. Values are means ( $n 3$ times), with standard errors represented by vertical bars. Mean value was significantly different from that of the male chow-fed group: ${ }^{* *} P<0.01,{ }^{* * *} P<0.001$. Mean value was significantly different from that of the female chow-fed group: † $P<0.05$, †† $P<0.01$, ††† $P<0.001$. 㧊 Mean value was significantly different from that of the male HFD-fed group $(P<0.001)$. Two-way ANOVA was performed to assess the effects of diet, sex and of their interaction (diet $\times$ sex) on ALP activity $(P<0.0001, P=0.0008$ and NS, respectively), the Alizarin assay $(P=0.0002, P=0.0003$ and NS, respectively) and the Oil red-O assay $(P<0.0001, P<0.0001$ and NS, respectively). OD, optical density. 
Table 6. Messenger RNA levels in long bones

(Mean values for eight mice per group with their standard errors)

\begin{tabular}{|c|c|c|c|c|c|c|c|c|c|c|c|}
\hline & \multicolumn{4}{|c|}{ Female } & \multicolumn{4}{|c|}{ Male } & & & \\
\hline & \multicolumn{2}{|c|}{ Chow } & \multicolumn{2}{|c|}{ HFD } & \multicolumn{2}{|c|}{ Chow } & \multicolumn{2}{|c|}{ HFD } & \multicolumn{3}{|c|}{ Two-way ANOVA: P§ } \\
\hline & Mean & SE & Mean & SE & Mean & SE & Mean & SE & Diet & Sex & Interaction \\
\hline Col-1 & 1 & 0.24 & 0.68 & 0.07 & 1 & 0.07 & $0 \cdot 13^{\star \star}+\dagger$ & 0.005 & 0.0022 & NS & NS \\
\hline RUNX-2 & 1 & 0.14 & $2 \cdot 10^{*} \dagger$ & 0.43 & 1 & 0.15 & $0.52^{\star \star}$ & 0.029 & NS & NS & 0.0119 \\
\hline $\mathrm{OCN}$ & 1 & 0.22 & $0.32^{\star *}+\dagger$ & 0.01 & 1 & 0.058 & $0.52^{\star \star}+\dagger$ & 0.001 & 0.0002 & NS & NS \\
\hline PPAR- $\gamma$ & 1 & 0.03 & 0.82 & $0 \cdot 10$ & 1 & 0.188 & $1.98^{\star * \star}+\dagger$ & $0 \cdot 10$ & 0.0105 & 0.0014 & NS \\
\hline aP-2 & 1 & 0.04 & 0.87 & 0.18 & 1 & 0.42 & $6 \cdot 47^{\star \star \star}+\dagger \dagger$ & 0.03 & $<0.0001$ & $<0.0001$ & $<0.0001$ \\
\hline TRAP & 1 & $0 \cdot 10$ & $1 \cdot 34$ & 0.17 & 1 & 0.198 & $2.97^{\star * \star}+\dagger \dagger$ & 0.17 & 0.0001 & 0.0013 & 0.0013 \\
\hline RANK & 1 & 0.16 & $1 \cdot 17$ & 0.51 & 1 & 0.024 & $21 \cdot 7^{\star \star \star}+\dagger \dagger$ & 0.65 & $<0.0001$ & $<0.0001$ & $<0.0001$ \\
\hline OPG/RANKL & 1 & 0.09 & 0.92 & 0.02 & 1 & 0.04 & $0.66^{\star}$ & 0.07 & NS & NS & NS \\
\hline
\end{tabular}

HFD, high-fat diet; Col-1, collagen type-1; RUNX-2, runt-related transcription factor 2; OCN, osteocalcin; aP-2, adipocyte protein-2; TRAP, tartrate-resistant acid phosphatase;

RANK, receptor activator of NF-kB; OPG/RANKL, osteoprotegerin/receptor activator of NF-kB ligand.

Mean value was significantly different from that of the male chow-fed group: ${ }^{*} P<0.05,{ }^{\star \star} P<0.01,{ }^{\star \star \star} P<0.001$.

Mean value was significantly different from that of the female chow-fed group: $\dagger P<0.05$, $\dagger+P<0.01,+\dagger \dagger P<0.001$.

$\S$ Values of two-way ANOVA are $P$ values for the main effects for the four groups of diet, and sex, and interaction between diet and sex.

regulate osteoclastogenesis and osteoclast function. We next studied whether or not the HFD modulates osteoblast function such that osteoclastogenesis is inhibited. When data were expressed as the ratio of OPG:RANKL (measure of induction of osteoclastogenesis), HFD treatment, specifically in males, led to a $30-40 \%$ decrease in the ratio. Altogether, the data suggest that HFD treatment for 10 weeks may lead to more deterioration in the bones of males as compared with females.

\section{Discussion}

In the present study we assessed the effects of association between HFD-induced osteoporosis in $\mathrm{C} 57 \mathrm{BL} / 6$ male and female mice. Obesity was induced by the HFD with $60 \%$ energy as fat in $\mathrm{C} 57 \mathrm{BL} / 6$ mice, as this strain is more likely to develop metabolic alterations that closely delineate the human metabolic syndrome such as dyslipidaemia, inflammation and obesity. Adiposity influences bone remodelling through secretion of several adipokines that directly target bone. These adipokines influence the central nervous system, thereby changing sympathetic impulses to bone and paracrine signals that influence adjacent skeletal cells ${ }^{(27,28)}$. Evidence from epidemiological and animal-feeding trials suggests that low vitamin $\mathrm{D}$ and/or $\mathrm{Ca}$ alone or along with a Western-style diet that is high in fat, low in Ca, vitamin D and folic acid may result in low bone mass and poor bone quality $^{(29,30)}$. Further, studies with high-fat high-sucrose diets or a diet low in folic acid led to abnormalities in bone ${ }^{(31-33)}$.

The present study with a HFD illustrates differential effects of HFD feeding in males and females. The HFD for 10 weeks increased body weight with deterioration of bone in young male mice as compared with females, but impairment in response to glucose challenge was found in females $v$. the males. The notion that obesity may not be beneficial to skeletal health is echoed frequently in paediatric literature and studies have shown that children and young adults with distal forearm fractures were more likely to have a higher degree of $\operatorname{adiposity}^{(34)}$. Further, sex differences have been suggested in humans in the prevalence of metabolic diseases, with males being more prone to the disease as compared with females ${ }^{(35)}$. Studies in human subjects show that there is differential accumulation of fat, fat mobilisation, utilisation as a fuel and finally the presence of excess and insufficient fat stores in both males and females. Many of these differences have evolved because of the differences in the much higher reproductive cost due to lactation and gestation in females as compared with males. Other studies have shown that NEFA release in men is higher because of higher subcutaneous fat deposits than women, making them resistant to anti-lipolytic effects of meal ingestion; therefore, regional differences in the regulation of fatty acid metabolism between men and women ${ }^{(36,37)}$ cannot be ignored. Studies also show that visceral fat depots with an inflammatory element secrete cytokines, including resistin, TNF- $\alpha$, IL- 1 and IL- 6 , which uncouple bone remodelling by enhancing bone resorption ${ }^{(27,28)}$. In osteoporosis too, these differences stand out prominently. Testosterone in contrast with oestrogen is not uniformly associated with fracture risk in men ${ }^{(38)}$. Androgen receptor-mediated action is important for trabecular bone maintenance in male mice whereas both oestrogens and androgens regulate cortical bone growth ${ }^{(39)}$. Further, bone mass that is an established determinant of bone strength is achieved at the end of the growth period, commonly designated as PBM. PBM plays an important role in the risk of osteoporotic fractures occurring in adulthood which significantly differs in males and females ${ }^{(40)}$. It is considered that a decrease of PBM by 1 SD may increase the fracture risk by $50 \%{ }^{(40)}$

We specifically investigated changes in both bone quality and content in the presence of a HFD in both males and females. Although larger bone size is expected, especially with higher body mass, mechanical performance of the bone was degraded by the effects of obesity with no change in bone size (length). Multiple comparisons between groups showed that diet was a major contributor to lipid changes observed in both sexes but its effects on bone were differential. Male animals on the HFD lost more trabecular bone as compared with the females. This was evident with higher urinary CTx (breakdown products of collagen type 1 
cross-linked C-telopeptide) levels in males $v$. females. We observed impaired glucose tolerance in female mice with unaltered plasma OCN levels while their male counterparts fed with the HFD remained protected towards glucose intolerance with modestly increased plasma OCN levels. Karsenty's group has shown that serum levels of uncarboxylated OCN regulate bone resorption mediated by insulin signalling in osteoblasts $^{(41)}$. Lower serum uncarboxylated OCN levels were observed in patients with osteoporosis taking medication that inhibits bone resorption, than in controls ${ }^{(41,42)}$. However, treatment for the duration of 10 weeks could be one of the limitations of the present study, as it led to no change in mechanical properties of the cortical bone compartment. However, maintenance of cortical bone because of the action of both the gonadal hormones (testosterone and oestrogen) as protection to the bone specifically in males at the cortical site cannot be ruled out.

Our data on bone marrow progenitor cells show preferential differentiation of these cells and genes more towards an adipogenic lineage as compared with osteogenic. In fact, haematopoietic cells experienced more osteoclastogenic response in the presence of the HFD. Studies show that more (number) marrow adipocytes as seen by us in HFD treatment exert a lipotoxic effect on osteoblast function and survival through the reversible biosynthesis of fatty acids into the bone marrow micro-environment ${ }^{(43)}$. Our data corroborate these findings, with a higher BMSC response towards adipogenesis. Thus, it is possible that it is the lipotoxic effect induced by adipocytes on osteoblast survival that may have contributed to cancellous bone loss in males as compared with females.

Overall, our data suggest that HFD feeding leads to a greater loss of bone and prevents preservation of the trabecular micro-architecture in males that may further increase fracture risk beyond bone mineral density.

\section{Supplementary material}

To view supplementary material for this article, please visit http://dx.doi.org/10.1017/S0007114514000051

\section{Acknowledgements}

The authors thank Swasti Tiwari for critical reading of the manuscript.

We gratefully acknowledge the University Grant Commission (UGC), Council of Scientific and Industrial Research (CSIR), Indian Council of Medical Research (ICMR), New Delhi, India for the award of research fellowships (to J. G., V. K., P. K., D. C. and R. S. S.). Financial support from 'BSC0103 (New approaches towards understanding disease dynamics and accelerate drug discovery)' is acknowledged. BSC0103 had no role in the design, analysis or writing of this article.

J. G. and D. C. contributed to the planning and execution of the animal experimental groups. J. G. conducted the biochemical analysis and gene expression studies. R. S. S. and D. C. carried out the oral glucose tolerance test experiment. V. K. and P. K. along with J. G. contributed to the cell culture and microcomputed tomography analyses. R. T. and D. S. contributed to the preparation of the manuscript. R. T. and S. T. provided the concept and resources for the experiments, and were involved in the planning, execution and troubleshooting of the experiments.

There are no conflicts of interest.

\section{References}

1. Rosen CJ \& Bouxsein ML (2006) Mechanisms of disease: is osteoporosis the obesity of bone? Nat Clin Pract Rheumatol 2, 35-43.

2. Rosen CJ, Beamer WG \& Donahue LR (2001) Defining the genetics of osteoporosis: using the mouse to understand man. Osteoporos Int 12, 803-810.

3. Kawai M, Devlin MJ \& Rosen CJ (2009) Fat targets for skeletal health. Nat Rev Rheumatol 5, 365-372.

4. Cao JJ, Gregoire BR \& Gao H (2009) High-fat diet decreases cancellous bone mass but has no effect on cortical bone mass in the tibia in mice. Bone 44, 1097-1104.

5. Ma H, Turpeinen T, Silvennoinen M, et al. (2011) Effects of diet-induced obesity and voluntary wheel running on the microstructure of the murine distal femur. Nutr Metab (Lond) 8, 1.

6. Cao JJ (2011) Effects of obesity on bone metabolism. J Orthop Surg Res 6, 30.

7. Sheu Y \& Cauley JA (2011) The role of bone marrow and visceral fat on bone metabolism. Curr Osteoporos Rep 9 , $67-75$.

8. Hsu YH, Venners SA, Terwedow HA, et al. (2006) Relation of body composition, fat mass, and serum lipids to osteoporotic fractures and bone mineral density in Chinese men and women. Am J Clin Nutr 83, 146-154.

9. Zhao LJ, Liu YJ, Liu PY, et al. (2007) Relationship of obesity with osteoporosis. J Clin Endocrinol Metab 92, 1640-1646.

10. Xiao Y, Cui J, Li YX, et al. (2011) Dyslipidemic high-fat diet affects adversely bone metabolism in mice associated with impaired antioxidant capacity. Nutrition 27, 214-220.

11. Lu XM, Zhao H \& Wang EH (2013) A high-fat diet induces obesity and impairs bone acquisition in young male mice. Mol Med Rep 7, 1203-1208.

12. Gerbaix M, Metz L, Mac-Way F, et al. (2012) Impact of an obesogenic diet program on bone densitometry, micro architecture and metabolism in male rat. Lipids Health Dis 11, 91.

13. Willinghamm MD, Brodt MD, Lee KL, et al. (2010) Agerelated changes in bone structure and strength in female and male BALB/c mice. Calcif Tissue Int 86, 470-483.

14. Xiao Y, Cui J, Li YX, et al. (2010) Expression of genes associated with bone resorption is increased and bone formation is decreased in mice fed a high-fat diet. Lipids 45, 345-355.

15. Gautam J, Kushwaha P, Swarnkar G, et al. (2012) EGb 761 promotes osteoblastogenesis, lowers bone marrow adipogenesis and atherosclerotic plaque formation. Phytomedicine 19, $1134-1142$.

16. Trivedi R, Kumar A, Gupta V, et al. (2009) Effects of Egb 761 on bone mineral density, bone microstructure, and osteoblast function: possible roles of quercetin and kaempferol. Mol Cell Endocrinol 302, 86-91.

17. Khedgikar V, Gautam J, Kushwaha P, et al. (2012) A standardized phytopreparation from an Indian medicinal plant (Dalbergia sissoo) has antiresorptive and bone-forming effects on a postmenopausal osteoporosis model of rat. Menopause 19, 1336-1346.

18. Malaval L, Modrowski D, Gupta AK, et al. (1994) Cellular expression of bone-related proteins during in vitro 
osteogenesis in rat bone marrow stromal cell cultures. $J$ Cell Physiol 158, 555-572.

19. Trivedi R, Kumar S, Kumar A, et al. (2008) Kaempferol has osteogenic effect in ovariectomized adult Sprague-Dawley rats. Mol Cell Endocrinol 289, 85-93.

20. Gupta GK, Kumar A, Khedgikar V, et al. (2013) Osteogenic efficacy enhancement of kaempferol through an engineered layer-by-layer matrix: a study in ovariectomized rats. Nanomedicine (Lond) 8, 757-771.

21. Khedgikar V, Kushwaha P, Gautam J, et al. (2013) Withaferin A: a proteasomal inhibitor promotes healing after injury and exerts anabolic effect on osteoporotic bone. Cell Death Dis 4, e778.

22. Heim M, Johnson J, Boess F, et al. (2002) Phytanic acid, a natural peroxisome proliferator-activated receptor (PPAR) agonist, regulates glucose metabolism in rat primary hepatocytes. FASEB J 16, 718-720.

23. Santiago-Mora R, Casado-Diaz A, De Castro MD, et al. (2011) Oleuropein enhances osteoblastogenesis and inhibits adipogenesis: the effect on differentiation in stem cells derived from bone marrow. Osteoporos Int 22, 675-684.

24. Wang XL, Suzuki R, Lee K, et al. (2009) Ablation of ARNT/ HIF1 $\beta$ in liver alters gluconeogenesis, lipogenic gene expression, and serum ketones. Cell Metab 9, 428-439.

25. Livak KJ \& Schmittgen TD (2001) Analysis of relative gene expression data using real-time quantitative PCR and the $2^{-\Delta \Delta C}$ T method. Methods 25, 402-408.

26. Kumar A, Gupta GK, Khedgikar V, et al. (2012) In vivo efficacy studies of layer-by-layer nano-matrix bearing kaempferol for the conditions of osteoporosis: a study in ovariectomized rat model. Eur J Pharm Biopharm 82, 508-517.

27. Karsenty G \& Oury F (2010) The central regulation of bone mass, the first link between bone remodeling and energy metabolism. J Clin Endocrinol Metab 95, 4795-4801.

28. Kawai M, de Paula FJ \& Rosen CJ (2012) New insights into osteoporosis: the bone-fat connection. J Intern Med 272, 317-329.

29. Ward WE, Kim S \& Bruce WR (2003) A Western-style diet reduces bone mass and biomechanical bone strength to a greater extent in male compared with female rats during development. Br J Nutr 90, 589-595.
30. Creedon A \& Cashman KD (2001) The effect of calcium intake on bone composition and bone resorption in the young growing rat. BrJ Nutr 86, 453-459.

31. Cavadini C, Siega-Riz AM \& Popkin BM (2000) US adolescent food intake trends from 1965 to 1996. Arch Dis Child $\mathbf{8 3}$ $18-24$.

32. Krumdieck CL \& Prince CW (2000) Mechanisms of homocysteine toxicity on connective tissues: implications for the morbidity of aging. J Nutr 130, 365S-368S.

33. Wohl GR, Loehrke L, Watkins BA, et al. (1998) Effects of high-fat diet on mature bone mineral content, structure, and mechanical properties. Calcif Tissue Int 63, 74-79.

34. Goulding A, Jones IE, Taylor RW, et al. (2001) Bone mineral density and body composition in boys with distal forearm fractures: a dual-energy X-ray absorptiometry study. J Pediatr 139, 509-515.

35. Vlassoff C (2007) Gender differences in determinants and consequences of health and illness. J Health Popul Nutr 25, 47-61

36. Power ML \& Schulkin J (2008) Sex differences in fat storage, fat metabolism, and the health risks from obesity: possible evolutionary origins. Br J Nutr 99, 931-940.

37. Blaak E (2001) Gender differences in fat metabolism. Curr Opin Clin Nutr Metab Care 4, 499-502.

38. Sinnesael M, Claessens F, Boonen S, et al. (2013) Novel insights in the regulation and mechanism of androgen action on bone. Curr Opin Endocrinol Diabetes Obes 20, $240-244$

39. Moverare S, Venken K, Eriksson AL, et al. (2003) Differential effects on bone of estrogen receptor $\alpha$ and androgen receptor activation in orchidectomized adult male mice. Proc Natl Acad Sci U S A 100, 13573-13578.

40. Bonjour JP, Chevalley T, Ferrari S, et al. (2009) The importance and relevance of peak bone mass in the prevalence of osteoporosis. Salud Publica Mex 51, Suppl. 1, S5-S17.

41. Lee NK, Sowa H, Hinoi E, et al. (2007) Endocrine regulation of energy metabolism by the skeleton. Cell 130, 456-469.

42. Ferron M, Wei J, Yoshizawa T, et al. (2010) Insulin signaling in osteoblasts integrates bone remodeling and energy metabolism. Cell 142, 296-308.

43. Elbaz A, Wu X, Rivas D, et al. (2010) Inhibition of fatty acid biosynthesis prevents adipocyte lipotoxicity on human osteoblasts in vitro. J Cell Mol Med 14, 982-991. 